\author{
Luiz Sérgio Silva ${ }^{\mathrm{a}}$ \\ (iD) https://orcid.org/0000-0002-3517-6941 \\ Elaine Leandro Machado ${ }^{a}$ \\ (iD) https://orcid.org/0000-0002-3226-3476 \\ Helian Nunes de Oliveira ${ }^{a}$ \\ (iD) https://orcid.org/0000-0003-1635-5175 \\ Adalgisa Peixoto Ribeiro ${ }^{a}$ \\ (iD) https://orcid.org/0000-0001-9415-8068
}

a Universidade Federal de Minas Gerais (UFMG), Faculdade de Medicina,

Departamento de Medicina Preventiva e Social. Belo Horizonte, MG, Brasil.

Contato:

Luiz Sérgio Silva

E-mail:

luizsergios1@gmail.com

Os autores declaram que o trabalho não foi subvencionado e que não há conflitos de interesses.

Os autores informam que o trabalho não foi apresentado em eventos científicos e que não foi baseado em dissertação ou tese.

\section{Condições de trabalho e falta de informações sobre o impacto da COVID-19 entre trabalhadores da saúde}

\author{
Working conditions and lack of information on the impact of \\ COVID-19 among health workers
}

\section{Resumo}

Introdução: diante da pandemia da COVID-19, torna-se importante rever questões de proteção da saúde dos trabalhadores. Objetivo: discutir as condições de saúde e segurança dos trabalhadores que cuidam de pacientes com COVID-19, sob a perspectiva das informações levantadas por seus representantes de classe profissional e de recomendações institucionais. Métodos: levantamento de informações na literatura científica, em documentos e orientações de entidades oficiais de saúde, em fontes de entidades sindicais e de representação de classes de profissionais de saúde. Discussão: começamos pela descrição das características da infecção pelo SARS-CoV-2 no processo de trabalho em saúde; exemplificamos as iniciativas de organizações representativas dos trabalhadores para o enfrentamento da COVID-19; descrevemos o cenário do trabalho em saúde na pandemia no Brasil; apresentamos o relato das medidas de proteção e de enfrentamento da doença orientadas por entidades e organismos nacionais e internacionais. Finalizamos discutindo que a exposição desses trabalhadores pode levar a outros eventos em saúde, necessitando medidas de adequação em relação a número de profissionais, melhoria na organização e nas condições de trabalho, fornecimento de equipamentos de proteção individual em quantidade e qualidade adequadas e implantação de medidas que propiciem o fortalecimento das equipes para o enfrentamento da COVID-19.

Palavras-chave: trabalhadores da saúde; COVID-19; equipamentos de proteção individual; saúde do trabalhador.

\begin{abstract}
Introduction: the Covid-19 pandemic has made it more relevant to review workers' health protection matters. Objective: to discuss the health and safety conditions of the health workers who care for COVID-19 patients, from the perspective of the information gathered by their professional class representatives and from institutional recommendations. Methods: we searched the academic literature, documents and guidelines provided by official health institutions and health workers' unions. Discussion: we described the characteristics of the infection by SARS-CoV-2 in health care work, presented initiatives taken by health workers' unions against the disease, outlined the health care working conditions during the pandemic in Brazil, and approached the protection measures recommended by national and international entities. We end by discussing how the exposure to these working conditions may lead the health workers to experience other clinical events that require compliance measures concerning the amount of professionals, improvement in organization and in working conditions, provision of adequate personal protective equipment, and implementation of measures to strengthen health teams to face COVID-19.
\end{abstract}

Keywords: health personnel; COVID-19; personal protective equipment; occupational health. 


\section{Introdução}

A emergência sanitária relacionada à pandemia da COVID-19 no Brasil e no mundo traz a necessidade de repensarmos velhas questões de proteção da saúde dos trabalhadores, sejam elas individuais ou coletivas.

A exposição ocupacional foi relacionada a um alto percentual dos primeiros casos de COVID-19, que ocorreram no primeiro epicentro da pandemia, Wuhan (China), tendo um mercado de frutos do mar como possível origem da contaminação, através do manuseio de animais vivos ${ }^{1}$.

À medida que o novo coronavírus (SARS-CoV-2) se espalha globalmente, cresce também a importância da saúde pública na caracterização do papel do ambiente e do processo de trabalho na transmissão da doença, dada a variedade de atividades que podem promover e facilitar sua disseminação, como verificado em epidemias e pandemias anteriores ${ }^{2}$. Os ambientes de trabalho podem ter um papel relevante na disseminação do vírus e, portanto, a análise de como se organizam é determinante para a prevenção do adoecimento. A compreensão de como os diferentes grupos ocupacionais estão expostos a infecções e doenças no local de trabalho pode ajudar nas respostas e no gerenciamento de riscos para a COVID-19 pela saúde pública e os subsequentes surtos de outras doenças infecciosas ${ }^{3}$.

Com o aumento do número de casos e a exigência dos cuidados de saúde para os casos graves, os profissionais da área da saúde foram reconhecidos como grupo de alto risco para adquirir a infecção ${ }^{4}$; como eles estão na linha de frente da resposta ao surto de COVID-19, estão expostos a fatores que os colocam em maior vulnerabilidade para o contágio e o adoecimento. Os riscos incluem exposição a patógenos, longas horas de trabalho, sofrimento psicológico, fadiga, esgotamento profissional, estigma e violência física e psicológica ${ }^{5}$.

A COVID-19 pode ser considerada a primeira nova doença relacionada ao trabalho a ser descrita nesta década, mostrando a importância prática do controle das infecções nos ambientes de trabalho, não apenas para profissionais de saúde, mas para a proteção de todos os grupos de trabalhadores envolvidos no cuidado e na assistência à população ${ }^{4}$.

Autores como Huh ${ }^{6}$ e Bahl et al. ${ }^{7}$ destacam que, além da prevenção do contágio dos trabalhadores, devem ser consideradas sua segurança física, suas condições de trabalho e sua estabilidade emocional e psíquica, motivando inclusive um gerenciamento para esses desafios no contexto da pandemia de COVID- $19^{8}$.
Nesse sentido, o objetivo deste ensaio é discutir as condições de saúde e segurança dos trabalhadores que cuidam de pacientes com COVID-19, sob a perspectiva das informações levantadas por seus representantes de classe profissional e de recomendações institucionais. Essa discussão é necessária para que não fiquem invisíveis esses profissionais que lidam diariamente com o atendimento à população brasileira nas unidades do Sistema Único de Saúde (SUS), sem ignorar que situações semelhantes, e até mesmo piores, possam estar acontecendo no setor privado.

\section{Métodos}

Foram realizados levantamentos de informações sobre a situação dos trabalhadores da saúde na pandemia da COVID-19 em publicações na literatura científica, em documentos e orientações de órgãos oficiais de saúde - como a Organização Mundial da Saúde (OMS), o Centers for Disease Control and Prevention (CDC), dos EUA, e o Ministério da Saúde do Brasil - e em outras fontes, como entidades sindicais e de representação de classes de profissionais de saúde. Além disso, realizou-se acompanhamento diário das informações publicadas sobre o tema nas principais mídias nacionais e internacionais, com verificação da consistência dos dados divulgados. Após esses levantamentos, procedeu-se à compilação das informações e à sua organização para composição do texto.

\section{Discussão}

\section{Características da infecção pelo novo coronavírus no processo de trabalho em saúde}

Em janeiro de 2020, a OMS divulgou que as autoridades chinesas tinham detectado um tipo de pneumonia causada pelo novo coronavírus, identificado em uma pessoa hospitalizada em Wuhan ${ }^{4}$. A OMS declarou Emergência de Saúde Pública de Importância Internacional (ESPII) por surto do novo coronavírus em 30 de janeiro de 2020. Em 12 de fevereiro de 2020, o novo vírus foi denominado como SARS-CoV-2, e a doença por ele causada de COVID-194,

Com base nas evidências disponíveis, o vírus SARS-CoV-2 é transmitido entre pessoas através de contato próximo e gotículas respiratórias produzidas quando uma pessoa infectada tosse ou espirra, bem como por superfícies contaminadas com o vírus. As pessoas com maior risco de infecção são aquelas que estão em contato próximo ou que cuidam de pacientes com COVID-19 ${ }^{10}$. No entanto, dados mais 
recentes indicam a possibilidade de transmissão aérea do SARS-CoV-2, pois existem evidências de sua viabilidade em aerossóis por pelo menos três horas ${ }^{11}$.

A exposição a esse agente configura "exposição biológica" e a maioria dos profissionais de saúde (se não todos) que atendem um paciente contaminado ficam sujeitos a um alto risco de adquirir a doença, particularmente durante a realização de procedimentos $^{12}$, embora inúmeros profissionais de outras áreas de atendimento à população neste momento crítico também estejam expostos ${ }^{13}$.

Pelas situações vivenciadas em outros países que enfrentaram a crise de assistência à população acometida pela epidemia de COVID-19, nota-se claramente a intensidade e a alta prevalência da infecção entre os trabalhadores da saúde. Segundo editorial da revista The Lancet $^{14}$, há relatos de prevalência superior a $10 \%$ nessa população, chegando a $20 \%$ entre os italianos. $\mathrm{O}$ texto relata ainda que cerca de 3.300 trabalhadores da saúde se infectaram na China e que aproximadamente 22 haviam morrido até o final de fevereiro de 2020, dentre os quais estava o profissional que alertou sobre o início da epidemia ${ }^{14}$.

Ampliando a visão do impacto da pandemia entre trabalhadores da saúde, a instituição italiana Federazione Nazionale degli Ordini dei Medici Chirurghi e degli Odontoiatri ${ }^{15}$ publicou em seu site, em 13 de maio de 2020, uma lista de 167 médicos que vieram a óbito em virtude de exposição, contágio e adoecimento pela COVID-19 até então, número esse atualizado diariamente.

O CDC ${ }^{16}$ apresentou a descrição preliminar dos indivíduos acometidos pela COVID-19 nos Estados Unidos da América em 17 de abril de 2020, na qual mostrou que 9.202 foram identificados como trabalhadores da saúde, com ocorrência de 27 mortes entre eles - óbitos registrados no período situado entre 12 de fevereiro e 9 de abril de 2020 . Heinzerling et al. ${ }^{17}$ documentaram, em fevereiro de 2020, os três primeiros casos de contaminação ocupacional nos EUA, deixando claro que se trata de uma doença altamente contagiosa e relacionada à exposição no trabalho. No Brasil houve necessidade do Supremo Tribunal Federal (STF) discutir e reconhecer que a COVID-19 pode ser considerada uma doença ocupacional ${ }^{18}$. A Medida Provisória ${ }^{0} 927 / 2020$, que autoriza medidas excepcionais para manter o vínculo empregatício durante a pandemia da COVID-19 e que não a reconhece como doença ocupacional, salvo em casos comprovados ${ }^{19}$, torna-se um grande problema para enquadrar os casos de contaminação e adoecimento entre os trabalhadores, expostos principalmente em seus locais de atuação diária, no atendimento à população, seja na atenção à saúde ou em outros tipos de prestação de serviços, agravando ainda mais sua situação desprotegida e vulnerável por ficarem sem cobertura em eventuais afastamentos por COVID-19.

Iniciativas de organizações representativas dos trabalhadores para enfrentamento da COVID-19

O Brasil conta com uma legislação abrangente que inclui as recomendações de órgãos internacionais sobre as ações de enfrentamento à COVID-19. No entanto, identificam-se lacunas no que se refere à fiscalização das medidas de segurança e saúde no trabalho. No tocante aos riscos, observam-se entendimentos equivocados sobre a exposição aos riscos de sofrer agravos ocupacionais, naturalizando-os em determinadas atividades, como se fossem aceitáveis. Neste sentido, a saúde do trabalhador é analisada de forma completamente à parte das condições de trabalho $^{20}$, não merecendo preocupação por parte dos gestores - que consideram treinamentos e equipamentos de proteção individual (EPIs) como suficientes, embora fornecidos em quantidade e com qualidade duvidosas.

O Sindicato dos Médicos de Minas Gerais ${ }^{21}$ emitiu guia informativo sobre as condições necessárias para a conduta médica no atendimento aos pacientes portadores da síndrome respiratória causada pela COVID-19, basicamente voltado para o uso de EPIs e para condições ambientais e de conforto no trabalho, abrindo um canal para denúncias através de e-mail e aplicativo de mensagens.

Na mesma linha, o Conselho Federal de Medicina $(\mathrm{CFM})^{22}$, em mensagem expedida aos médicos brasileiros no dia 30 de março de 2020, alertou sobre a obrigatoriedade do fornecimento de EPIs aos profissionais que estão na linha de frente de combate à pandemia de COVID-19, bem como criou, através de um link especial, um fórum para denúncias de descumprimento dessa obrigatoriedade nas condições de trabalho. O Conselho Federal de Enfermagem $(\text { Cofen })^{23}$ foi na mesma direção dessas exigências de condições adequadas de trabalho, expressas na necessidade de EPIs.

No entanto, somente o Cofen ${ }^{23}$ está realizando buscas no sentido de obter estatísticas de profissionais a ele filiados acometidos e/ou mortos em decorrência da COVID-19. As abordagens dos dois conselhos de profissionais da saúde (médicos e enfermeiros) levam em conta as situações de trabalho denunciadas diariamente através de diversas mídias, embora se limitem a apresentar a legislação que remete ao uso de EPIs e à possibilidade de recusa ao trabalho sob condições de grave risco à saúde e à vida. Cabe ressaltar que os EPIs, em situações emergenciais, diante da gravidade do quadro do paciente e da alta contagiosidade do agente de risco biológico, principalmente quando a organização e as condições 
de trabalho não permitem que estas sejam controladas, podem não proteger de maneira adequada o trabalhador exposto, além de não existirem provas da efetividade plena desses equipamentos ${ }^{7,24}$.

O Comitê Gestor de Crise do Cofen lançou, no dia 6 de maio de 2020, o site Observatório da Enfermagem, com atualizações constantes sobre a evolução da COVID-19 entre os profissionais, indicando que já são quase 17 mil enfermeiros, técnicos e auxiliares de enfermagem afastados pela doença no Brasil, com 138 óbitos associados à doença ${ }^{25}$. O Cofen pontua que essas mortes já são mais que o dobro do número absoluto registrado entre profissionais de enfermagem na Itália, primeiro epicentro da pandemia no Ocidente, retratando o impacto das infecções dessa pandemia entre enfermeiros, técnicos e assistentes ${ }^{25}$.

\section{Cenário do trabalho em saúde na pandemia da COVID-19 no Brasil}

Nos últimos anos, a emergência e a reemergência de doenças infecciosas suscitaram muitas questões sobre o papel da vigilância em saúde nos sistemas de saúde, pois colocam à prova a capacidade da vigilância e da assistência quanto à oportunidade de detecção precoce e ao poder de resposta. No que tange à vigilância em saúde do trabalhador, no contexto desta pandemia, a necessidade de proteção dos profissionais dos estabelecimentos de saúde ganhou o merecido destaque; entretanto, carecemos de bases de dados ou levantamentos oficiais que retratem os impactos da COVID-19 na saúde desses trabalhadores. Uma das fontes de dados mais importantes para essas informações seria a ficha de notificação da COVID-19 do Ministério da Saúde, que, entretanto, não incorporou dados mais específicos sobre trabalho, especificando apenas as ocupações dos trabalhadores notificados e, mais recentemente, da segurança pública ${ }^{26}$.

As informações oficiais de morbimortalidade têm sido divulgadas pelos vários níveis de gestão do SUS, estratificadas por sexo, faixa etária e região geográfica, contribuindo para orientar medidas de controle e prevenção, planejamento e alocação de recursos. No entanto, não há estratificação por ocupação, por exemplo, variável fundamental para analisar as condições de trabalho de profissionais que têm resultado positivo no teste da doença e essa falta de indicadores dificulta a identificação de focos de disseminação relacionados com as atividades laborais ${ }^{14}$.

Entre os profissionais de saúde brasileiros, a divulgação dos contaminados, dos acometidos pela doença e dos óbitos relativos à exposição à COVID19 tem sido feita basicamente pela mídia e por representantes de classe. Outros profissionais praticamente não são lembrados.
As dificuldades na obtenção de dados oficiais sobre o número de profissionais de saúde afastados por suspeita de COVID-19 em todo o Brasil foram apontadas pelo portal $G 1^{27}$, que apurou, em pesquisa realizada entre 12 e 18 de abril de 2020, um total aproximado de 7 mil profissionais (médicos, enfermeiros e técnicos de enfermagem) afastados do trabalho desde o início da pandemia. O Ministério da Saúde ${ }^{28}$ divulgou, em 18 de maio de 2020, a morte de 113 médicos em decorrência da doença, compondo uma média de quase dois óbitos por dia desde 16 de março de 2020, quando a primeira morte foi registrada. No mesmo período, o Brasil contava 31.790 casos confirmados da doença entre profissionais de saúde, e mais 114 mil casos em investigação. $O G l o b o^{29}$ realizou um levantamento junto às representações dos médicos (Conselhos Regionais de Medicina e sindicatos) no qual indica que cerca de $30 \%$ dos óbitos (27 casos) correspondiam a profissionais do grupo de risco para a doença, com idade superior a 60 anos. Considerando-se que nem todas as entidades consultadas responderam, é possível que esse número seja ainda maior.

O Brasil é ainda o país onde mais morrem enfermeiros. Segundo nota do dia 28 de maio de 2020, o Cofen e o Conselho Internacional de Enfermagem (ICN, na sigla em inglês) afirmam que houve 157 mortes de profissionais de enfermagem (enfermeiros, técnicos e auxiliares), número maior que o dos Estados Unidos (146 óbitos) e do Reino Unido (77 óbitos). Naquela data, havia 5,5 mil casos confirmados nessa categoria profissional ${ }^{30}$.

Em Belo Horizonte (MG), dentre os 1.994 profissionais de saúde submetidos ao teste da COVID-19, 115 tiveram resultado positivo $(5,8 \%)^{31}$. Pensando na necessidade de expansão da testagem de profissionais de saúde, mesmo que estes não tenham sintomas da doença, a Assembleia Legislativa de Minas Gerais aprovou, no dia 21 de maio de 2020, projeto de lei que determina que profissionais da saúde e da segurança pública passem por teste de COVID-19 a cada 15 dias $^{32}$.

É importante uma ação mais efetiva quanto à vigilância da saúde do trabalhador para acompanhar os impactos da COVID-19 na saúde desses profissionais que estão na linha de frente do combate à doença. Quanto aos boletins epidemiológicos dos vários níveis de gestão do SUS, seria importante haver, minimamente, a divulgação da distribuição dos casos e dos óbitos confirmados segundo as ocupações registradas.

\section{Medidas de proteção e enfrentamento da COVID-19 adotadas pelos sistemas de saúde}

Pesquisadores ${ }^{14}$ relataram que, à medida que a epidemia avança, o acesso aos EPIs se torna objeto de 
preocupação em todo o mundo. A equipe médica é priorizada em muitos países, mas a escassez de EPIs é uma constatação nos locais mais afetados. Algumas equipes médicas aguardam o equipamento enquanto já atendem pacientes que podem estar infectados, ou são fornecidos equipamentos que podem não atender aos requisitos mínimos de proteção. A situação no Brasil não é diferente, havendo relatos diários dessas situações em diversas mídias.

Outro pesquisador, $\mathrm{Huh}^{6}$, afirmou que a segurança dos trabalhadores da saúde é essencial para que eles ofereçam os melhores serviços profissionais possíveis para as pessoas infectadas. Ele relatou ainda o aumento da carga de trabalho entre enfermeiros através do prolongamento das jornadas de trabalho. Essa sobrecarga de trabalho foi decorrente da escassez de médicos e de outros profissionais de saúde para cuidar do grande volume de pacientes. Os profissionais de saúde passaram então a enfrentar um recrudescimento da carga de trabalho, associado à possibilidade de serem expostos e infectados pelo SARS-CoV-2.

É evidente a crise dos sistemas de saúde mundiais, incapazes de dar conta de uma epidemia de tamanhas proporções e que pode levar à hospitalização cerca de $15 \%$ a $20 \%$ dos $\operatorname{casos}^{33,34}$. Cabe perguntar: quando esses profissionais adoecerem, quem vai cuidar da população em geral adoecida, da qual eles também farão parte, então? E o que será desses trabalhadores e de suas famílias? Continuarão amargurados e expostos à infecção sem as devidas condições de trabalho? Quem cuidará deles? São questões absolutamente sem respostas adequadas no presente momento.

Uma reportagem do jornal inglês The Guardian ${ }^{35}$ de 18 de abril de 2020, intitulada "NHS staff told 'wear aprons' as protective gowns run out', indicou a informação de que os chefes do National Health System (NHS) britânico pediram que médicos e enfermeiros trabalhassem sem avental de proteção no tratamento de pacientes da COVID-19, já que faltavam suprimentos nos hospitais. Isso infringe as orientações de saúde pública emitidas pelo próprio NHS, que estipulam que aventais cirúrgicos à prova d'água de comprimento total e demais equipamentos deveriam ser usados para todos os procedimentos hospitalares de alto risco - o que pode ser exemplo do que acontece em todo o mundo neste momento de emergência sanitária. No Brasil não é diferente. Os profissionais reclamam diariamente nas mídias da falta de EPIs, e os gestores relatam estar apenas gerindo o uso consciente e racional destes. Além do risco em si, o medo se torna um companheiro indissociável da população de trabalhadores da saúde, particularmente daqueles da enfermagem.

Uma publicação do jornal Le Monde ${ }^{36}$, de 18 de abril de 2020, seguiu a linha de abordagem da perda de trabalhadores na batalha contra a COVID-19, informando que conhecer o número de profissionais de saúde doentes ou falecidos por COVID-19 na França é um verdadeiro obstáculo, porque nas estatísticas sobre a propagação da pandemia, no mundo e na França, a questão dos médicos, enfermeiros e auxiliares de enfermagem contaminados quase nunca é abordada. O mesmo se verifica na Espanha, nos EUA e por todo o mundo. Os principais jornais mundiais deixaram de publicar notícias sobre os trabalhadores da saúde após as mudanças nas fases da epidemia em seus países, com redução dos casos novos, das internações em unidades de terapia intensiva e das mortes diárias. É um esquecimento inaceitável em face dos trabalhadores da saúde, tidos como heróis há menos de um mês ${ }^{37}$.

Para fazer frente à disseminação da pandemia do novo coronavírus, as políticas assistenciais devem levar em conta ações de saúde pública voltadas para os grupos com maior vulnerabilidade à contaminação em decorrência de sua atividade de trabalho, entre eles os trabalhadores da saúde, que podem ter um papel relevante na dinâmica de disseminação do vírus; deve-se analisar seu processo e condições de trabalho para entender seu papel na disseminação do agente ${ }^{3}$, bem como seu modo de se contaminar, adoecer e morrer em decorrência dessa exposição. A não observância desse aspecto implica sua invisibilidade e pouca valorização nas políticas públicas voltadas para sua proteção ${ }^{14}$, levando ao adoecimento e óbito de milhares de trabalhadores.

Segundo Chow et al. ${ }^{38}$, as intervenções para impedir a transmissão da COVID-19 pelos trabalhadores da saúde incluem a triagem sorológica baseada em critérios de sintomatologia expandida, o afastamento e a melhoria do acesso ao teste pelos sintomáticos, e a criação de políticas de licença médica sem caráter punitivo, mais flexíveis e consistentes com as orientações de saúde pública. Os mesmos autores ${ }^{38}$ relataram que o uso de EPIs por todos os profissionais de saúde pode auxiliar na redução da transmissão por pacientes assintomáticos ou levemente sintomáticos. Isso pode ser particularmente importante em ambientes ambulatoriais, instituições de longa permanência e regiões com ampla transmissão comunitária. Deve-se ainda acrescentar a redução da transmissão horizontal entre trabalhadores e entre estes e as pessoas com quem mantêm contato diário, inclusive seus familiares.

\section{Considerações finais}

No contexto de uma pandemia como a da COVID-19, as abordagens tradicionais da saúde e da segurança no trabalho, assim como outras sustentadas nos pressupostos do campo da saúde do trabalhador, precisavam ser repensadas e adaptadas ao novo cenário 
de crise. Nesse sentido, medidas de adequação da equipe em relação a números, melhoria na organização e nas condições de trabalho, fornecimento de EPIs em quantidade e qualidade adequadas, com treinamento também adequado de uso e descarte, são urgentes e necessárias ${ }^{39}$. A observação de normativos e diretrizes desenvolvidas por organismos e instituições como a OMS, o CDC, o Centers for Medicare $\&$ Medicaid Services (CMS), entre outros, voltados ao controle da COVID-19 entre os trabalhadores da saúde na linha de frente de atendimento aos pacientes pode melhorar muito esse painel atual. No Brasil, o Ministério da Saúde e os conselhos de classe de médicos e enfermeiros também emitiram recomendações nesse sentido. As chefias de equipe precisam ser proativas, sendo importante fortalecer o grupo, usar de franqueza e discutir conjuntamente as situações a serem enfrentadas.

Também cumpre lembrar outras morbidades que podem afetar a saúde dos trabalhadores da saúde expostos às condições de trabalho para enfrentamento da pandemia por COVID-19, entre elas os problemas de saúde mental, como burnout, depressão, fadiga mental, transtornos psiquiátricos menores e até morte por sobrecarga de trabalho, conhecida como karoshi. A previsão inicial é de mais ondas na pandemia, inclusive uma de adoecimento mental, trazendo um sério temor do surgimento de sequelas mentais incapacitantes e do aumento das taxas de suicídio entre trabalhadores da saúde ${ }^{40}$.

\section{Contribuições de autoria}

Silva LS participou da concepção do estudo, do levantamento e da análise dos resultados, da elaboração do manuscrito, da revisão crítica e da aprovação da versão a ser publicada. Machado EL, Oliveira HN e Ribeiro AP participaram da elaboração do estudo, do levantamento e da análise dos resultados, da elaboração do manuscrito, da revisão crítica e da aprovação da versão final a ser publicada. Todos os autores assumem integral responsabilidade pelo trabalho publicado.

\section{Referências}

1. Li Q, Guan X, Wu P, Wang X, Zhou L, Tong Y, et al. Early transmission dynamics in Wuhan, China, of novel coronavirus-infected pneumonia. $\mathrm{N}$ Engl J Med [Internet]. 2020 [citado em 4 abr 2020];382:1199-207. https://doi.org/10.1056/ NEJMoa2001316

2. Edwards $\mathrm{CH}$, Tomba GS, de Blasio BF. Influenza in workplaces: transmission, workers' adherence to sick leave advice and European sick leave recommendations. Eur J Public Health [Internet]. 2016 [citado em 14 maio 2020];26(3):478-85. https://doi.org/10.1093/eurpub/ckw031

3. Baker MG, Peckham TK, Seixas NS. Estimating the burden of United States workers exposed to infection or disease: a key factor in containing risk of COVID-19 infection. medRxiv [Internet]. 6 mar 2020 [citado em 14 maio 2020]. https://doi. org/10.1101/2020.03.02.20030288

4. Koh D. Occupational risks for COVID-19 infection. Occup Med [Internet]. 2020 [citado em 24 maio 2020];70(1):3-5. https://doi.org/10.1093/ occmed/kqaa036

5. Coronavirus disease (COVID-19) outbreak: rights, roles and responsibilities of health workers, including key considerations for occupational safety and health: interim guidance. World Health Organization [Internet]. 18 mar 2020 [citado em 5 abr 2020]. Disponível em: https://www.who.int/ publications-detail/coronavirus-disease-(COVID19)-outbreak-rights-roles-and-responsibilities-of- health-workers-including-key-considerations-foroccupational-safety-and-health

6. Huh S. How to train health personnel to protect themselves from SARS-CoV-2 (novel coronavirus) infection when caring for a patient or suspected case. J Educ Eval Health Prof [Internet]. 2020 [citado em 5 maio 2020];17:10. https://doi. org/10.3352/jeehp.2020.17.10

7. Bahl P, Doolan C, de Silva C, Chughtai AA, Bourouiba L, MacIntyre CR. Airborne or droplet precautions for health workers treating coronavirus disease 2019? J Infect Dis [Internet]. 2020 [citado em 5 maio 2020];jiaa189. http://doi.org/10.1093/ infdis/jiaa189

8. Greenberg N, Docherty M, Gnanapragasam S, Wessely S. Managing mental health challenges faced by healthcare workers during covid-19 pandemic. BMJ [Internet]. 26 mar 2020 [citado em 5 maio 2020];368:m1211. https://doi.org/10.1136/ bmj.m1211

9. Statement on the second meeting of the International Health Regulations (2005) Emergency Committee regarding the outbreak of novel coronavirus (2019-nCoV). World Health Organization [Internet]. 30 jan 2020 [citado em 5 abr 2020]. Disponível em: https://www.who. int/news-room/detail/30-01-2020-statementon-the-second-meeting-of-the-internationalhealth-regulations-(2005)-emergency-committeeregarding-the-outbreak-of-novel-coronavirus(2019-ncov) 
10. Rational use of personal protective equipment for coronavirus disease (COVID-19) and considerations during severe shortages: interim guidance. World Health Organization [Internet]. 6 abr 2020 [citado em 10 maio 2020]. Disponível em: https://www.who.int/publications/i/item/ rational-use-of-personal-protective-equipment-forcoronavirus-disease-(covid-19)-and-considerationsduring-severe-shortages

11. Correia G, Rodrigues L, Gameiro da Silva M, Gonçalves T. Airborne route and bad use of ventilation systems as non-negligible factors in SARS-CoV-2 transmission. Med Hypotheses [Internet]. 25 abr 2020 [citado em 10 maio 2020];141:109781. http://doi.org/10.1016/j. mehy.2020.109781

12. Ferioli M, Cisternino C, Leo V, Pisani L, Palange P, Nava S. Protecting healthcare workers from SARS-CoV-2 infection: practical indications. Eur Respir Rev [Internet]. 2020 [citado em 10 maio 2020];29(155):200068. http://doi. org/10.1183/16000617.0068-2020

13. Jackson Filho JM, Assunção AA, Algranti E, Garcia EG, Saito CA, Maeno M. A saúde do trabalhador e o enfrentamento da COVID-19. Rev Bras Saude Ocup [Internet]. 2020 [citado em 9 maio 2020];45:e14. https://doi.org/10.1590/23176369ed0000120

14. COVID-19: protecting health-care workers. Lancet [Internet]. 2020 [citado em 10 maio 2020];395(10228):922. https://doi.org/10.1016/ S0140-6736(20)30644-9

15. Elenco dei Medici caduti nel corso dell'epidemia di Covid-19. Federazione Nazionale degli Ordini dei Medici Chirurghi e degli Odontoiatri [Internet]. 2020 [citado em 3 jun 2020]. Disponível em: https://portale.fnomceo.it/elenco-dei-medicicaduti-nel-corso-dellepidemia-di-COVID-19/

16. CDC COVID-19 Response Team. Characteristics of Health Care Personnel with COVID-19: United States, February 12-April 9, 2020. MMWR Morb Mortal Wkly Rep [Internet]. 2020 [citado em 20 abr 2020];69(15):477-81. http://dx.doi.org/10.15585/ mmwr.mm6915e6

17. Heinzerling A, Stuckey MJ, Scheuer T, Xu K, Perkins KM, Resseger H, et al. Transmission of COVID-19 to Health care personnel during exposures to a hospitalized patient: Solano County, California, February 2020. MMWR Morb Mortal Wkly Rep [Internet]. 2020 [citado em 13 maio 2020];69(15):472-6. http://dx.doi.org/10.15585/ mmwr.mm6915e5

18. Saraiva R. STF reconhece Covid como acidente de trabalho. Agora São Paulo [Internet]. 4 maio 2020 [citado em 13 maio 2020]. Disponível em: https:/agora.folha.uol.com.br/grana/2020/05/stfreconhece-covid-como-acidente-de-trabalho.shtml

19. Brasil. Medida Provisória $\mathrm{n}^{\circ}$ 927, de 22 de março de 2020. Dispõe sobre as medidas trabalhistas para enfrentamento do estado de calamidade pública reconhecido pelo Decreto Legislativo $\mathrm{n}^{\circ}$ 6, de 20 de março de 2020, e da emergência de saúde pública de importância internacional decorrente do coronavírus (covid-19), e dá outras providências. Diário Oficial da União. 22 mar 2020;158(55-L):1-3.

20. Galon T, Marziale MHP, Souza WL. A legislação brasileira e as recomendações internacionais sobre a exposição ocupacional aos agentes. Rev Bras Enferm [Internet]. 2011 [citado em 10 maio 2020];64(1):160-167. http://dx.doi.org/10.1590/ S0034-71672011000100023

21. Sindicato dos Médicos de Minas Gerais. Pandemia COVID-19: guia de orientações da conduta do médico [Internet]. Belo Horizonte: Sinmed-MG; 2020 [citado em 10 maio 2020]. Disponível em: https://sinmedmg.org.br/sinmed-mg-lancaguiade-orientacoes-da-conduta-do-medico-pandemiaCOVID-19/

22. Combate à COVID-19: CFM divulga orientações para o trabalho dos médicos durante o período de enfrentamento do coronavírus. Conselho Federal de Medicina [Internet]. 20 mar 2020 [citado em 19 abr 2020]. Disponível em: http://portal.cfm.org. $\mathrm{br} /$ index.php?option $=$ com_content $\&$ view $=$ article $\&$ id=28641:2020-03-20-22-16-32\&catid=3

23. Informação de Profissionais de Enfermagem com COVID-19 [Internet]. 2020 [citado em 19 abr 2020]. Disponível em: https://docs.google. com/forms/d/e/1FAIpQLSd_UTZBDglkMU4H 7r0jErSSWo6o3YSZ4O4AT_5RHD5Xa1vTdw/ viewform? $\mathrm{vc}=0 \& \mathrm{c}=0 \& \mathrm{w}=1$

24. Agalar C, Öztürk Engin D. Protective measures for COVID-19 for healthcare providers and laboratory personnel. Turk J Med Sci [Internet]. 2020 [citado em 5 maio 2020];50:578-84. http://doi.org/10.3906/ sag-2004-132

25. Cofen registra 10 mil casos suspeitos de COVID-19 entre profissionais de Enfermagem. Conselho Federal de Enfermagem [Internet]. 6 maio 2020 [citado em 13 maio 2020]. Disponível em: http:// www.cofen.gov.br/cofen-registra-10-mil-casos-decovid-19-entre-profissionais-de-enfermagem_79551. html\#: :text=Cofen\%20registra\%2010\%20 mil\%20casos\%20de\%20COVID\%2D19\%20 entre\%20profissionais\%20de\%20Enfermagem,-Observat\%C3\%B3rio\%20da\%20Enfermagem\&text=O\%20Comit\%C3\%AA\%20Gestor\%20 de\%20Crise,COVID\%2D19\%20entre\%20os\%20 profissionais.

26. A Covid-19, a saúde e bem estar dos trabalhadores: a barbárie entre nós. Associação Brasileira de Saúde Coletiva [Internet]. 28 abr 2020 [citado em 24 maio 2020]. Disponível em: https://www. abrasco.org.br/site/noticias/sistemas-de-saude/acovid-19-a-saude-e-bem-estar-dos-trabalhadores-abarbarie-entre-nos/47465/

27. Covid-19 faz vítimas entre profissionais da saúde no Brasil. G1 [Internet]. 12 abr 2020 [citado em 19 abr 2020]. Disponível em: https://g1.globo.com/ fantastico/noticia/2020/04/12/covid-19-faz-vitimasentre-profissionais-da-saude-no-brasil.ghtml 
28. Brasil, Ministério da Saúde, Secretaria Nacional de Vigilância em Saúde. Boletim Epidemiológico Especial COE-COVID19 [Internet]. Brasília: Ministério da Saúde; 18 maio 2020 [citado em 2 jun 2020]. 67 p. Boletim n. 16. Disponível em: https:// www.saude.gov.br/images/pdf/2020/May/21/202005-19---BEE16---Boletim-do-COE-13h.pdf

29. Schmitt G. Brasil ultrapassa a marca de cem médicos mortos por Covid-19, dois por dia. O Globo [Internet]. 21 maio 2020 [citado em 2 jun 2020]. Disponível em: https://oglobo.globo. com/sociedade/coronavirus/brasil-ultrapassamarca-de-cem-medicos-mortos-por-COVID-19dois-por-dia-1-24438369

30. Brasil é o país com mais mortes de enfermeiros por Covid-19 no mundo. Conselho Federal de Enfermagem [Internet]. 28 maio 2020 [citado em 28 maio 2020]. Disponível em: http://www.cofen.gov.br/ brasil-e-o-pais-com-mais-mortes-de-enfermeiros-porCOVID-19-no-mundo-dizem-entidades_80181.html

31. Prefeitura Municipal de Belo Horizonte, Secretaria de Saúde. Notificações de COVID-19. Boletim Epidemiológico [Internet]. 1 jun 2020 [citado em 2 jun 2020];30. Disponível em: https://prefeitura. pbh.gov.br/sites/default/files/estrutura-de-governo/ saude/2020/imagens/boletim_epidemiologico_30_ covid-19_01-06-2020.pdf

32. Paiva D. Covid-19: ALMG aprova projeto que obriga testagem em profissionais da saúde e da segurança a cada 15 dias. G1 [Internet]. 21 maio 2020 [citado em 2 jun 2020]. Disponível em: https:/g1.globo.com/mg/minas-gerais/ noticia/2020/05/21/covid-19-almg-aprova-projetoque-obriga-testagem-em-profissionais-da-saude-eda-seguranca-a-cada-15-dias.ghtml

33. Freitas ARR, Napimoga M, Donalisio MR. Análise da gravidade da pandemia de Covid-19. Epidemiol Serv Saude [Internet]. 2020 [citado em 14 maio 2020];29(2):e2020119. https://doi.org/10.5123/ s1679-49742020000200008

34. Moghadas SM, Shoukat A, Fitzpatrick MC, Wells CR, Sah P, Pandey A, et al. Projecting hospital utilization during the COVID-19 outbreaks in the United States. Proc Natl Acad Sci U S A [Internet]. 2020 [citado em 14 maio 2020];117(16):9122-6. https://doi. org/10.1073/pnas.2004064117

35. Campbell D. NHS staff told 'wear aprons' as protective gowns run out. The Guardian [Internet]. 17 abr 2020 [citado em 19 abr 2020]. Disponível em https://www.theguardian.com/world/2020/ apr/17/nhs-staff-to-be-asked-to-treat-coronaviruspatients-without-gowns

36. Barroux R. Coronavirus: le difficile décompte des morts des soignants en France. Le Monde [Internet]. 17 abr 2020 [citado em 18 abr 2020]. Disponível em: https://www.lemonde.fr/ sante/article/2020/04/17/en-france-le-difficilecomptage-du-tribut-paye-par-les-soignants-aucovid-19_6036872_1651302.html

37. Kuper S. How health workers replaced soldiers as society's heroes. Financial Times [Internet]. 25 mar 2020 [citado em 9 maio 2020]. Disponível em: https://www.ft.com/content/03b82e0c-6e3711ea-9bca-bf503995cd6f

38. Chow EJ, Schwartz NG, Tobolowsky FA, Zacks RLT, Huntington-Frazier M, Reddy SC, Rao AK. Symptom screening at illness onset of health care personnel with SARS-CoV-2 infection in King County, Washington. JAMA [Internet]. 17 abr 2020 [citado em 14 maio 2020];323(20):2087-9. https://doi.org/10.1001/jama.2020.6637

39. Gallasch CH, Cunha ML, Pereira LAS, Silva-Júnior JS. Prevenção relacionada à exposição ocupacional do profissional de saúde no cenário de COVID-19. Rev Enferm UERJ [Internet]. 2020 [citado em 9 maio 2020];28:e49596. http://dx.doi.org/10.12957/ reuerj.2020.49596

40. Troyer EA, Kohn JN, Hong S. Are we facing a crashing wave of neuropsychiatric sequelae of COVID-19? Neuropsychiatric symptoms and potential immunologic mechanisms. Brain Behav Immun [Internet]. 13 abr 2020 [citado em 13 maio 2020];87:34-9. http://doi.org/10.1016/j. bbi.2020.04.027 